\title{
Molecular characterization of the giant freshwater prawn (Macrobrachium rosenbergii) beta-actin gene promoter
}

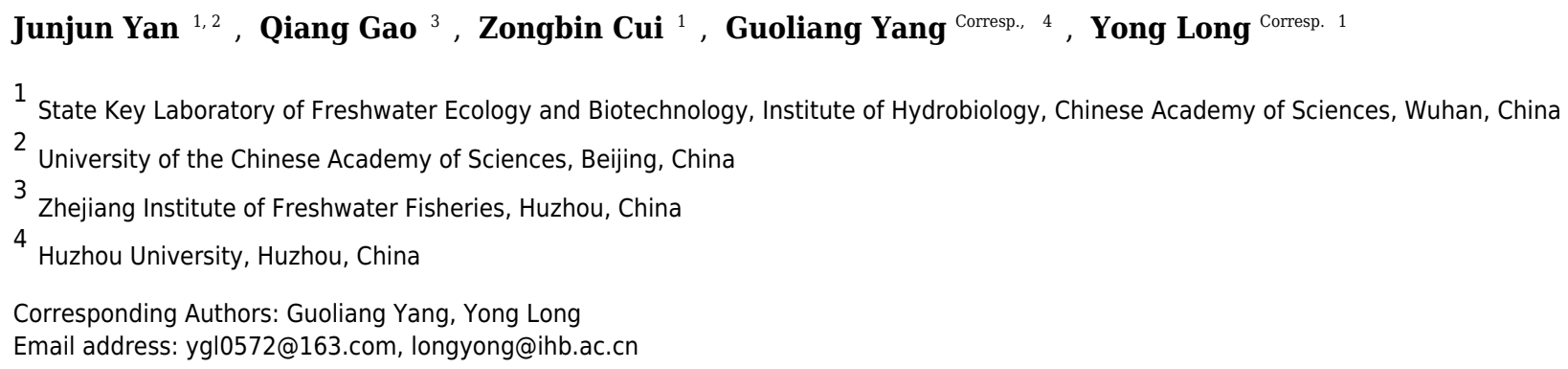

Constitutive promoters are important tools for gene function studies and transgenesis. The Beta-actin (actb1) gene promoter has been isolated from many species but remains to be cloned from the giant freshwater prawn (Macrobrachium rosenbergii) . In this study, we cloned and characterized the Mractb1 gene promoter. Two alternative promoters were identified for the Mractb1 gene, which direct the generation of two transcripts with different 5' untranslated region. Three CpG islands were predicted in the upstream sequence, which are intimately related to transcription initiation and promoter activity. In addition to the CCAAT-box and CArG-box, molecular dissection of the flanking sequence revealed the existence of one negative and two positive elements in the upstream region and the first intron. Finally, the Mractb1 promoter demonstrated comparative activity to the carp (Cyprinus carpio) actb1 promoter. Our investigations provide a valuable genetic tool for gene function studies and shed light on the regulation of the Mractb1 gene. 
1 Molecular characterization of the giant freshwater prawn (Macrobrachium

2 rosenbergii) beta-actin gene promoter

3

4 Junjun Yan $^{1,2}$, Qiang $\mathrm{Gao}^{3}$, Zongbin Cui ${ }^{1}$, Guoliang Yang ${ }^{4 *}$, Yong Long ${ }^{1^{*}}$

5

$6{ }^{1}$ State Key Laboratory of Freshwater Ecology and Biotechnology, Institute of Hydrobiology,

7 Chinese Academy of Sciences, Wuhan 430072, China

8

$9 \quad{ }^{2}$ University of the Chinese Academy of Sciences, Beijing, China

10

11

${ }^{3}$ Zhejiang Institute of Freshwater Fisheries, Huzhou, China

12

13

${ }^{4}$ Huzhou University, Huzhou, China

14

$15 *$ Corresponding authors

16 Yong Long ${ }^{1}$

17 longyong@ihb.ac.cn (YL)

18 Guoliang Yang ${ }^{4}$

19 yg10572@163.com (GY)

20

21

22

23

24

25 
26 Abstract

27 Constitutive promoters are important tools for gene function studies and transgenesis. The Betaactin (actb1) gene promoter has been isolated from many species but remains to be cloned from the giant freshwater prawn (Macrobrachium rosenbergii). In this study, we cloned and characterized the Mractbl gene promoter. Two alternative promoters were identified for the Mractb1 gene, which direct the generation of two transcripts with different $5^{\prime}$ untranslated region.

Three $\mathrm{CpG}$ islands were predicted in the upstream sequence, which are intimately related to transcription initiation and promoter activity. In addition to the CCAAT-box and CArG-box, molecular dissection of the flanking sequence revealed the existence of one negative and two positive elements in the upstream region and the first intron. Finally, the Mractb1 promoter demonstrated comparative activity to the carp (Cyprinus carpio) actb1 promoter. Our investigations provide a valuable genetic tool for gene function studies and shed light on the regulation of the Mractbl gene.

\section{Introduction}

41 Giant freshwater prawn is the biggest freshwater prawn in the world. The males can grow up to $320 \mathrm{~mm}$ in body length and weigh over $200 \mathrm{~g}$ (Ling 1969). It has a wide distribution throughout the world and is particularly favored for farming in the tropical and subtropical developing countries in Asia, such as China, India and Thailand. Owing to its fast growth rate and higher profit realization per unit area, it now has become the most widely aquacultured freshwater prawn species and contributed $51.7 \%$ to the total global production of freshwater prawns (Banu 
47 \& Christianus 2016; New 2005; New \& Nair 2012). However, outbreak of diseases is a major

48 limitation facing aquaculture industry and several diseases such as white spot disease and white

49 tail disease have been reported to cause mass mortality for giant freshwater prawn farming

50 worldwide (Bonami \& Widada 2011; Saurabh \& Sahoo 2008).

52 Transgenic technology has been considered as a powerful and effective way to improve

53 economic traits of aquaculture species (Bennett \& Jennings 2013; Rasmussen \& Morrissey 2007;

54 Sin 1997), through which pathogen-resistant strains of prawn could be generated to reduce or

55 even avoid the disease-related economic losses. So far, successful gene transfer in freshwater

56 prawn and several marine shrimp species have been reported (Arenal et al. 2000; Liu et al. 2001;

57 Preston et al. 2000). However, most of the researches used expression vectors based on

58 heterologous promoters such as CMV (cytomegalovirus) and SV40 (simian vacuolating virus 40)

59 promoter, hobo transposable element (HFL1) and carp beta-actin gene promoter. Studies about

60 fish transgenesis have shown that promoters of fish origin were more efficient in driving

61 expression of the target genes than those from mammalian or viral origins (Alam et al. 1996;

62 Hanley et al. 1998). Moreover, negative concerns about the use of viral promoters to express

63 transgenes have led to the generation of "all-fish" transgene constructs (Rasmussen \& Morrissey

64 2007). In accordance with the "all-fish" concept, promoters originated from prawn species have

65 strong potential for use in prawn transgenesis and gene function studies.

67 Beta-actin is a housekeeping gene with ubiquitous expression. The promoter of beta-actin gene 
68

69

70

71

72

has been isolated from and characterized in a wide variety of species such as human (Gunning et al. 1987), chicken (Kosuga et al. 2000), amphioxus (Feng et al. 2014), teleosts (Barman et al. 2015; Hwang et al. 2003; Kong et al. 2014; Liu et al. 1991; Noh et al. 2003) and shrimps (Shi et al. 2015; Shi et al. 2016). Because beta-actin gene promoter confers high level of constitutive transcriptional expression, it is widely used for transgenesis in both plants and animals (Cho et al. 2011; Hong et al. 2016; Kosuga et al. 2000). Although the cDNA of the beta-actin gene has been cloned from giant freshwater prawn (Zhu et al. 2005), its promoter remains to be characterized. Here, we report the isolation and characterization of the giant freshwater prawn beta-actin (Mractb1) gene promoter (Mbap). Our results provide insights into the regulation mechanisms of Mractb1 gene and indicate the potential usage of Mbap in gene function and transgenesis studies for giant freshwater prawn and closely related species.

\section{Materials and Methods}

\section{Animals}

The animal protocol of this study was approved by the Institutional Animal Care and Use Committee of Institute of Hydrobiology (Approval ID: Y341131501). Adult giant freshwater prawns of both sexes (body weight 20 - $30 \mathrm{~g}$ ) were acquired from Zhejiang Institute of Freshwater Fisheries, Huzhou, China. After transportation, the prawns were acclimated to laboratory conditions for 2 weeks in a circulating fresh water system at $28^{\circ} \mathrm{C}$. 


\section{Total RNA and genomic DNA extraction}

90

The prawns were immersed in eugenol solution $(0.125 \mathrm{~mL} / \mathrm{L}$ in Phosphate Buffered Saline) until loss of consciousness and then placed into ice-slurry for a 2-Step euthanasia procedure (Leary et al. 2013). After that their bodies were dissected and total RNA was extracted from the gill, liver, muscle and intestine of both sexes, and from testis and ovary, using TRIZOL (Invitrogen) according to the manufacturer's instructions. For genomic DNA extraction, about $100 \mathrm{mg}$ muscle tissue was put into a $1.5 \mathrm{~mL}$ Eppendorf tube with $0.5 \mathrm{~mL}$ lysis buffer $(10 \mathrm{mM}$ Tris-Cl, $1 \mathrm{mM}$ EDTA, $0.1 \%$ SDS, $200 \mu \mathrm{g} / \mathrm{mL}$ proteinase $\mathrm{K}, \mathrm{pH} 8.0$ ). The samples were incubated in $58^{\circ} \mathrm{C}$ water bath for 6 hours with gentle vibration. After that, the lysate was subjected to a traditional phenol/chloroform extraction procedure. The concentration of RNA and DNA samples was measured by a NanoDrop 8000 from Thermo Scientific and the quality of RNA and DNA samples was assessed by agarose gel electrophoresis.

\section{Genome walking}

Genome walking was performed as previously described (Zhou et al. 2015) to clone the 5' flanking sequence of the Mractbl gene. Each walking step contains three rounds of nested PCR using different gene specific primers (GSP). Before genome walking, primers Mractb1-F and Mractb1-R (sequences of all the primers used in this study are listed in Table 1) designed according to the cDNA sequence deposited in GenBank (AY626840) were used to amplify partial genomic sequence. A 1293-bp genomic DNA fragment was obtained and sequenced. The gene specific primers from Mractb1-GSP1 to Mractb1-GSP3 were subsequently designed 
110

111

112

113

114 115 116 117 118 119

according to this known genomic sequence. These gene specific primers were sequentially mated with the 4 degenerate primers included in the genome walking kit (Takara) to amplify the unknown 5' flanking sequence. Similarly, gene specific primers from Mractb1-GSP4 to Mractb1GSP6 based on the product of the first walking experiment were used for the second walking step. A total of 3 walking experiments were conducted to identify the $5^{\prime}$ flanking sequence of the Mractbl gene.

\section{5' RACE}

5' RACE (rapid amplification of cDNA ends) was performed using the Takara 5'-Full RACE Kit according to the manufacturer's instructions. Briefly, the RNA sample was first treated with CIAP (calf intestine alkaline phosphatase) to remove the naked phosphorous from the RNA molecules, followed by TAP (tobacco acid pyrophosphatase) treatment for mRNA decapitation.

The decapped mRNA was ligated to the 5' RACE adaptor using T4 RNA ligase. Reverse transcription was performed using M-MLV (moloney murine leukemia virus) reverse transcriptase and 9-mer random primer. After that, two rounds of nest PCR using 5'-RACEouter-primer/GSP1 and 5'-RACE-inner-primer/GSP2 primer pairs were conducted sequentially to clone the 5' cDNA ends. Product of the second round PCR was purified using the Biospin Gel Extraction Kit (BioFlux), subcloned into the pMD18-T vector (TaKaRa) and then sequenced by the Tsingke Biological Technology (Wuhan, China) Co., Ltd.

\section{Quantitative real-time PCR}


131

132

134

135

136

137

138

139

140

141

142

143

144

145

146

147

148

149

150

151

Quantitative real-time PCR (qPCR) was performed as previously described (Long et al. 2013) to determine the expression level of the Mractbl transcripts. Primers for qPCR were designed using the Primer Premier 6.0 software. First-strand cDNA was synthesized using total RNA extracted from different tissues by the RevertAid First Strand cDNA Synthesis Kit (Thermo scientific) and used as template for qPCR analysis. Mr $18 S-r R N A$ was used as the internal reference for gene expression normalization.

\section{Bioinformatic analyses}

$\mathrm{CpG}$ islands in the upstream region were predicted by the Methprimer software using default parameters (http://www.urogene.org). Core promoter elements were predicted by the YAPP Eukaryotic Core Promoter Predictor (http://www.bioinformatics.org/yapp/cgi-bin/yapp.cgi).

\section{Construction of plasmids}

To dissect transcriptional activity of the 5' flanking sequence of the Mractb1 gene, the cloned upstream sequences were divided into 7 fragments according to location of the predicted GC islands and the TSSs (transcriptional start sites) revealed by 5' RACE. The position of the first nucleotide of the initiation codon was regarded as +1 . The length and relative location of fragments 1 to 7 are 558 bp (-558 to -1$), 523 \mathrm{bp}$ (-1080 to -558), $240 \mathrm{bp}$ (-1317 to -1078), $249 \mathrm{bp}$ (-1568 to -1320), 195 bp (-1763 to -1569$), 357$ bp (-2120 to -1764$)$ and 501 bp (-2620 to -2120$)$, respectively. These fragments were amplified and cloned into the SacI/NheI site of the pGL3Basic vector (Promega) separately or in combination. Deletion of fragment 3 in the $5^{\prime}$ flanking 
152 15

154

155

156

157

158

159

160

161

162

163

164

165

166

167

168

169

170

171

172

sequences was conducted via the PCR-driven overlap extension method previously described

(Heckman \& Pease 2007) with primers Mractb1P42-F and Mractb1P24-R. The constructs were named according to the inserted fragments, for example, pGL-Mba7654321 includes the whole sequence, pGL-Mba1 contains only fragment 1 and pGL-Mba21 consists of both fragment 1 and fragment 2. To compare the promoter activity of Mractb1 gene with that of carp actb1 gene, the promoter of carp actb1 gene was amplified by the primer pair Carpactb1-F1/Carpactb1-R1 and inserted into the HindIII/NcoI site of the pGL3-Basic vector.

\section{Luciferase activity assay}

The EPC (endothelial progenitor cells) cells (ATCC ${ }^{\circledR} \mathrm{CRL}-2872^{\mathrm{TM}}$ ) were maintained at $28^{\circ} \mathrm{C}$ with 5\% $\mathrm{CO}_{2}$ in $\mathrm{M} 199$ medium (Hyclone) supplemented with $10 \%$ fetal bovine serum, $100 \mu \mathrm{g} / \mathrm{mL}$ streptomycin, $100 \mathrm{U} / \mathrm{mL}$ penicillin and $2 \mu \mathrm{g} / \mathrm{mL}$ amphotericin B. One day before transfection, cells were seeded into 24 -well plates at a density of $2 \times 10^{5}$ cells/well. Transfection was performed using the X-tremeGENE HP Transfection Reagent (Roche). The pRL-TK plasmid from Promega was used as internal control for luciferase activity assays and cotransfected with the promoter-luciferase constructs. The total amount of plasmid used for transfection was $500 \mathrm{ng} / \mathrm{well}$ and the ratio between the promoter-luciferase vector and pRL-TK was 5:1. Twenty-four hours after transfection, cells were lysed and subjected to luciferase activity assays using the Dual-Luciferase ${ }^{\circledR}$ Reporter Assay System from Promega.

\section{Western blot}


173 EPC cells cultured in 6-well plates were transfected with $2 \mu \mathrm{g}$ plasmid. Twenty-four hours after

174 transfection, cells were lysed by the RIPA (radio immunoprecipitation assay) lysis buffer from

175 beyotime. Then western blot was performed as previously described (Mo et al. 2010) and

176 GAPDH (glyceraldehyde-3-phosphate dehydrogenase) was used as loading control. The primary

177 antibodies used for western blot were rabbit anti-Firefly Luciferase (Abcam, \#ab185923, 1:5000)

178 and mouse anti-GAPDH (Boster, \#BM3876, 1:100). The secondary antibodies were goat anti-

179 rabbit IgG (immunoglobulin) (Boster, \#BM3894, 1:5000) and goat anti-mouse IgG (Boster,

180 \#BM3895, 1:5000), respectively. Intensity of the bands in the western blot image was analyzed

by the ImageJ software (http://rsb.info.nih.gov/ij/).

Statistical Analysis

The results of dual luciferase assays and qPCR are presented as mean \pm standard deviation.

Independent samples T tests were performed using SPSS (statistical product and service solutions) 15.0 to analyze the significant difference between groups.

Results

\section{Cloning of the 5' flanking sequence for the Mractb1 gene}

To clone the 5' flanking sequence for the Mractb1 gene, a 1293 bp genome sequence was firstly amplified using the primers Mractb1-F/ Mractb1-R designed according to the reported cDNA sequence (Zhu et al. 2005). Three rounds of genome walking experiments were subsequently 
194 195 196 197 198 199 200 201 202 203

performed to clone the upstream sequence of the Mractbl gene. As shown in (Fig. 1), the length of the DNA fragments obtained by the three genome walking experiments were 2094, 531 and 713 bp, respectively. Furthermore, PCR using the forward primer Mractb1-F and the reverse primer Exon3-R (matches to the 3' cDNA end) generated a 1593 bp sequence. Assembling of these fragments resulted in a 4306-bp long contig, which contains both the $5^{\prime}$ flanking and full sequence for the Mractb1 gene. The obtained upstream sequence counted from the first nucleotide of the initiation codon is $2620 \mathrm{bp}$ in length, which has been submitted to the GenBank database under the accession number KY038927.

\section{Identification of two transcripts for the Mractb1 gene}

Before characterizing the promoter of the Mractb1 gene, 5'-RACE was performed to identify the transcriptional start site (TSS). A clear DNA band in the range of 100 to $250 \mathrm{bp}$ was obtained

(Fig. 2). The DNA fragments were purified and cloned into the pMD18-T vector. Subsequent

DNA sequencing revealed two 5' cDNA end sequences for the Mractbl gene. One is 106 bp (10 clones sequenced) and the other is $145 \mathrm{bp}$ (6 clones sequenced) in length (Fig. 2). Further analysis revealed that the Mractb1 gene possesses 2 transcript variants (designated Mractb1_tv1 and Mractb1_tv2, respectively), which only differ in the 5' UTR (untranslated region sequence) (Fig. 3A). PCR using different forward primers (Exon1-F and Exon1'-F) and the same reverse primer (Exon3-R) amplified the full-length sequence for the 2 transcripts (Fig. 3A, B), which are 1186 and 1225 bp in length, respectively. Since the transcripts contain different exon 1 (Fig. 3A), we speculate that they are driven by alternative promoters (promoter-1 and promoter-2). 


\section{Expression of the Mractb1 transcripts in different sexes and tissues}

217 To shed light on the activities of the promoters in different sexes and tissues, the expression

218 levels of the 2 transcript variants were measured by qPCR assays. As shown in (Fig. 4), the

219 Mractb1 gene demonstrate quite different expression levels among tissues and between sexes.

220 The highest overall expression level of the Mractbl gene was found in the gonad and gill,

221 followed by the intestine, muscle and liver. Sexual dimorphism could be found for the expression

222 level of the Mractb1 gene in all the tissues (Fig. 4). The expression levels of both Mractb1_tv1

223 and Mractb1_tv2 in the female gonad (ovary) and gill is significantly higher than those in the

224 corresponding male tissues, while muscle and intestine of the males demonstrated higher

225 expression than those of the females (Fig. 4). Difference between the expression of the two

226 transcripts in same tissue was identified in the male gill and the female gonad and muscle.

227 Mractb1_tv2 showed higher expression in the male gill, but lower expression in the female

228 muscle and gonad when compared to Mractb1_tv1 (Fig. 4). These findings indicate that

229 expression of the Mractb1 gene is regulated by tissue and sex specific factors.

Molecular dissection of the Mractb1 gene promoter

Three CpG islands were predicted in the 5' flanking sequence of the Mractb1 gene. These CpG islands are located at -1762 to -1569 (i1, $194 \mathrm{bp}),-1437$ to -1320 (i2, $118 \mathrm{bp}$ ) and -747 to -542

234 (i3, 206 bp), respectively (Fig. 5A). The 2620 bp flanking sequence was divided into 7 segments 235 (from Mba 7 to Mba1) according to location of the CpG islands and the TSSs (Fig. 5A). 
236 Transcriptional activities of the upstream fragments were investigated by dual luciferase assays.

237 When characterized separately, Mba7, Mba6, Mba3 and Mba1 demonstrated no or minimal

238 transcriptional activity, while high promoter activity was detected for Mba5, Mba4 and Mba2,

239 each of them contains a predicted $\mathrm{CpG}$ island (Fig. 5B), suggesting importance of the CpG

240 islands in transcription. Based on structure of the Mractb1 transcripts and transcriptional

241 activities of the upstream fragments, we concluded that the two alternative promoters are in

242 Mba54 (promoter 1) and Mba21 (promoter 2), respectively. Activity of the two promoters and

243 regulations by their corresponding upstream regions were further characterized. For the

244 promoter-1, combination of Mba5 and Mba4 only slightly increased the promoter activity, Mba6

245 displayed no effect on Mba54, while fuse Mba7 to Mba654 significantly enhanced the promoter

246 activity (Fig. 5C). As for the promoter-2, significant synergistic effect was found between Mba2

247 and Mba1; however, Mba3 demonstrated negative effect on the activity of Mba21 (Fig. 5D).

248 Serial deletion experiments indicated that Mba654321 had the highest transcriptional activity,

249 followed by Mba7654321 and Mba54321, while Mba4321, Mba321 and Mba21 only displayed

250 basal promoter activity (Fig. 5E). Furthermore, interaction between the two alternative promoters

251 was observed. Mba54 (promoter-1) and Mba21 (promoter-2) demonstrated significant

252 synergistic effects when combined (Mba5421). Both Mba3 and Mba6 can enhance activity of

$253 \mathrm{Mba5421}$ and the interaction between Mba3 and Mba6 is necessary for the full activity of the

254 Mractb1 gene promoter (Fig 5F). Taken together, the upstream fragments demonstrate different

255 effects under different circumstances. For example, Mba7 enhances activity of the promoter-1

256 but inhibits activity of the whole promoter (Fig. 5C, E). On the contrary, Mba3 inhibits activity 
257 of the promoter-2 but is indispensable for the full activity of the whole promoter (Fig. 5D, F).

258 These findings suggest complex interactions among genetic elements in regulating expression of 259 the Mractbl gene.

260

261 Genetic elements in the Mractb1 gene promoter

262 As shown in (Fig. 5A, B), the CpG islands in the promoter of the Mractb1 gene are intimately 263 associated with the promoter activity. It's interesting that despite no TSS was identified in the 264 first $\mathrm{CpG}$ island (i1, Mba5), it still directs high level of transcription (Fig. 5B). Further investigation indicated that this region contains a CCAAT box, a CArG box and a GC box (Fig. 6). Both CCAAT box and CArG box were reported to be important for the constitutive expression of carp and human beta-actin gene (Liu et al. 1990; Quitschke et al. 1989). The second CpG island (i2) encompasses the first TSS and an initiator element (InR) (Fig. 6). The third CpG island (i3) contains the second TSS, a GC box and an InR (Fig. 6). Since no TATA box was identified in the core promoters for the Mractbl gene, the InRs may play important roles in transcription initiation.

\section{Mractb1 promoter demonstrates comparative activity with the carp actb1 promoter}

To justify whether the Mractbl promoter cloned in this study has the potential to be used as a genetic tool in gene function and transgenesis studies, the activity of which was compared with that of carp beta-actin (actbl) gene promoter, a promoter which is commonly used in transgenic fish. The fragment with the highest promoter activity (Mba654321) was designated as Mbap. 
278 The promoter of the carp $a c t b 1$ gene (designated as Cbap) was cloned into the pGL3- basic

279 vector as well. Dual luciferase assays in EPC cells indicated that the Mbap demonstrated

280 comparative activity with the Cbap (Fig. 7A). Western blot using antibody against the firefly

281 luciferase detected similar expression level of firefly luciferase in the EPC cells transfected with

282 pGL-Mbap and pGL-Cbap (Fig. 7B, C); however, the empty vector pGL-basic demonstrated no

283 luciferase expression at all. These results indicate that the Mractb1 promoter is as active as the

284 carp $a c t b 1$ promoter and which can be used for transgenesis and gene function studies for prawn 285 and related species in the future.

287 Discussion

As a house keeping gene, the promoter of $a c t b 1$ is cloned from a wide range of organisms and

extensively used for gene function and transgenesis studies (Barman et al. 2015; Cho et al. 2011;

Hwang et al. 2003; Kong et al. 2014; Liu et al. 1991; Noh et al. 2003). Although the CDS

(coding sequence) and 3' UTR sequence of the Mractbl gene have been cloned previously (Zhu

et al. 2005), the promoter remains to be characterized. In this study, we cloned the upstream sequence of the Mractb1 gene, identified the TSSs, investigated expression of the transcripts in different tissues of both sexes and characterized transcriptional activity of the Mractb1 gene promoter. Both luciferase assays and western blot indicated that the Mractb1 promoter demonstrated comparable transcriptional activity to that of the carp actbl promoter in the EPC cells, suggesting that it is a valuable tool for gene function and transgenesis studies for 
299

300

301

302

303

304

305

306

307

308

309

310

311

312

313

314

315

316

317

318

319

Macrobrachium rosenbergii.

Identification of two TSSs and the results of promoter activity assays indicated that the Mractb1 gene possesses two alternative promoters which initiate transcription from different TSSs and

lead to the generation of two transcript variants. These two transcript isoforms contain different initial exons but share the same open reading frame (ORF) and 3' UTR, indicating that they only differ in the 5' UTR. Existence of alternative promoters is common for mammalian genes. It was reported that $18 \%$ of human genes have evidence of alternative promoter usage (Landry et al. 2003). Alternative promoters may differ in tissue and developmental stage specificity and transcriptional activity (Landry et al. 2003). The usage of alternative promoters may account for distinct mRNA levels of the Mractb1 gene transcripts in different tissues and sexes, and different 5' UTR of the two transcript variants may affect their translation efficiency. Moreover, molecular dissection for the activities of different upstream regions revealed significant synergistic effects between the two alternative promoters. Although the actbl gene promoters of human and carp were well characterized, it is unknown whether they possess alternative promoters as well.

Three CpG islands were identified in the 5' flanking sequence of the Mractbl gene. These CpG islands are closely related to the activity of the Mractbl gene promoter according to luciferase assays. All of them demonstrated transcriptional activity and both of the identified TSSs are located in the two proximal $\mathrm{CpG}$ islands, consistent to the discovery that most $\mathrm{CpG}$ islands function as sites of transcription initiation by destabilizing nucleosomes and attracting proteins 
320

321

322

323

324

325

326

327

328

329

330

331

332

333

334

335

336

337

\section{9}

that create a transcriptionally permissive chromatin state (Deaton \& Bird 2011). CpG islands are prevalent in the promoter of housing keeping $(\mathrm{HK})$ genes and more than three quarters $(78.7 \%)$

of human HK (housing keeping) genes predominantly have a CpG+/TATA- core promoter (Zhu et al. 2008). CpG-islands usually contain multiple GC-boxes, and GC-boxes coupled with InR elements are essential to initiate transcription in the absence of TATA-box (Butler \& Kadonaga 2002). Consistent with this notion, both GC-box and InR, but no TATA-box were found in the core promoters of the Mractbl gene.

Genetic elements including the CCAAT-box and CArG-box were reported to be important for the constitutive expression of human and carp actb1 gene (Liu et al. 1990; Quitschke et al. 1989). These elements were also identified in the Mractb1 promoter. Fuse the DNA fragment containing these elements (Mba5) to the proximal promoter sequence (Mba4321) dramatically increased the promoter activity (Fig. 5A, E), suggesting the function of these elements in regulating the Mractbl gene. Like in the carp actbl gene promoter, a distal upstream region (Mba7) was found to negatively regulates the Mractb1 promoter activity. Moreover, the upstream fragment Mba6 and 5' end of the first intron Mba3, and the interaction between these two regions are necessary for the constitutive transcriptional activity of the Mractb1 gene promoter. These results indicate that in addition to the conservative elements, other unknown elements and factors are involved in regulating the Mractbl gene.

Conclusions 
341 The beta-actin (Mractb1) gene promoter of M. rosenbergii was cloned and characterized. Two

342 alternative promoters were identified for the Mractbl gene, which direct the generation of two

343 transcripts with different 5' UTR. Genetic dissection of the upstream sequence of the Mractb1

344 gene revealed one distal negative element and two proximal positive elements regulating the

345 activity of the Mractb1 gene promoter. Finally, the Mractb1 promoter demonstrated comparative

346 activity to the carp (Cyprinus carpio) $a c t b 1$ promoter. Our investigations provide a valuable

347 genetic tool for gene function studies and shed light on the regulation of the Mractbl gene.

349 Acknowledgements

350

351

352

353

354

355

356

357

358

359

360

361

362

363

364

365

366

367

368

The authors would like to thank Dr. Bolan Zhou from the Hunan university of arts and science

for technical assistance.

\section{References}

Alam MS, Lavender FL, Iyengar A, Rahman MA, Ayad HH, Lathe R, Morley SD, and Maclean N. 1996. Comparison of the activity of carp and rat $\beta$-actin gene regulatory sequences in tilapia and rainbow trout embryos. Molecular Reproduction \& Development 45:117.

Arenal A, Pimentel R, Guimarais M, Rodríguez A, Martínez R, and Abad Z. 2000. Gene Transfer in Shrimp (Litopenaeus schmitti) by Electroporation of Single-cell Embryos and Injection of Naked DNA into Adult Muscle. Biotecnologia Aplicada 17:247-250.

Banu R, and Christianus A. 2016. Giant Freshwater Prawn Macrobrachium rosenbergii Farming: A Review on its Current Status and Prospective in Malaysia. Journal of Aquaculture Research \& Development 7:423.

Barman HK, Mohanta R, Patra SK, Chakrapani V, Panda RP, Nayak S, Jena S, Jayasankar P, and Nandanpawar P. 2015. The $\beta$-actin gene promoter of rohu carp (Labeo rohita) drives reporter gene expressions in transgenic rohu and various cell lines, including spermatogonial stem cells. Cellular \& Molecular Biology Letters 20:237247.

Bennett DJ, and Jennings RC. 2013. Successful Agricultural Innovation in Emerging Economies: Transgenic marine algae for aquaculture: a coupled solution for protein sufficiency. Cambridge University Press, 233-246.

Bonami JR, and Widada JS. 2011. Viral diseases of the giant fresh water prawn Macrobrachium rosenbergii: A 
review. Journal of Invertebrate Pathology 106:131-142.

Butler JEF, and Kadonaga JT. 2002. The RNA polymerase II core promoter: a key component in the regulation of gene expression. Genes \& Development 16:2583-2592.

Cho YS, Sang YL, Kim YK, Dong SK, and Nam YK. 2011. Functional ability of cytoskeletal $\beta$-actin regulator to drive constitutive and ubiquitous expression of a fluorescent reporter throughout the life cycle of transgenic marine medaka Oryzias dancena. Transgenic Research 20:1333-1355.

Deaton AM, and Bird A. 2011. CpG islands and the regulation of transcription. Genes \& Development 25:10101022.

Feng J, Li G, Liu X, Wang J, and Wang YQ. 2014. Functional analysis of the promoter region of amphioxus betaactin gene: a useful tool for driving gene expression in vivo. Molecular Biology Reports 41:6817-6826.

Gunning P, Leavitt J, Muscat G, Ng SY, and Kedes L. 1987. A Human Beta-actin Expression Vector System Directs High-Level Accumulation of Antisense Transcripts. Proceedings of the National Academy of Sciences of the United States of America 84:4831-4835.

Hanley S, Smith TJ, Muller F, Maclean N, Uzbekova S, Prunet P, and Breton B. 1998. Isolation and functional analysis of the histone H3 promoter from atlantic salmon (Salmo salar L.). Molecular Marine Biology \& Biotechnology 7:165.

Heckman KL, and Pease LR. 2007. Gene splicing and mutagenesis by PCR-driven overlap extension. Nature Protocols 2:924.

Hong JK, Suh EJ, Kwon SJ, Lee SB, Jin AK, Lee SI, and Lee YH. 2016. Promoter of chrysanthemum actin confers high-level constitutive gene expression in Arabidopsis and chrysanthemum. Scientia Horticulturae 211:8-18.

Hwang G-L, Rahman MA, Razak SA, Sohm Fdr, Farahmand H, Smith A, Brooks C, and Maclean N. 2003. Isolation and characterisation of tilapia $\beta$-actin promoter and comparison of its activity with carp $\beta$-actin promoter. Biochimica et Biophysica Acta 1625:11-18.

Kong HJ, Kim JL, Kim WJ, Kim HS, Yeo SY, Park JY, and An CM. 2014. Genomic cloning and promoter analysis of the beta-actin gene from Korean rose bitterling (Rhodeus uyekii). Genes \& Genomics 36:861-869.

Kosuga M, Enosawa S, Li XK, Suzuki S, Matsuo N, Yamada M, Roychowdhury J, Koiwai O, and Okuyama T. 2000. Strong, long-term transgene expression in rat liver using chicken beta-actin promoter associated with cytomegalovirus immediate-early enhancer (CAG promoter). Cell Transplantation 9:675-680.

Landry JR, Mager DL, and Wilhelm BT. 2003. Complex controls: the role of alternative promoters in mammalian genomes. Trends in Genetics 19:640-648.

Leary S, Underwood W, Anthony R, Cartner S, Corey D, Grandin T, Greenacre CB, Gwaltney-Bran S, McCrackin MA, and Meyer R. 2013. AVMA Guidelines for the Euthanasia of Animals: 2013 Edition. University of Alaska Anchorage.

Ling SW. 1969. The general biology and development of the macrobrachium rosenbergii (de man). FAO Fisheries Report 57:589-606.

Liu Z, Xiang J, Zhou G, and Gong Z. 2001. Foreign gene transfer into Chinese shrimps (Penaeus chinensis) with gene gun. Chinese Science Bulletin 46:766-769.

Liu ZJ, Moav B, Faras AJ, Guise KS, Kapuscinski AR, and Hackett PB. 1990. Functional-Analysis of Elements Affecting Expression of the Beta-actin Gene of Carp. Molecular and Cellular Biology 10:3432-3440.

Liu ZJ, Zhu ZY, Roberg K, Faras A, Guise K, Kapuscinski AR, and Hackett PB. 1991. Isolation and characterization of beta-actin gene of carp (Cyprinus carpio). Mitochondrial DNA 1:125-136. 
410

411

412

413

414

415

416

417

418

419

420

421

422

423

424

425

426

427

428

429

430

431

432

433

434

435

436

437

438

439

440

441
Long Y, Song G, Yan J, He X, Li Q, and Cui Z. 2013. Transcriptomic characterization of cold acclimation in larval zebrafish. BMC gonomics 14:612.

Mo S, Wang L, Li Q, Li J, Li Y, Thannickal VJ, and Cui Z. 2010. Caveolin-1 regulates dorsoventral patterning through direct interaction with $\beta$-catenin in zebrafish. Developmental Biology 344:210-223.

New MB. 2005. Freshwater prawn farming: global status, recent research and a glance at the future. Aquaculture Research 36:210-230.

New MB, and Nair CM. 2012. Global scale of freshwater prawn farming. Aquaculture Research 43:960-969.

Noh JK, Cho KN, Han EH, Kim AR, Lee JS, Dong SK, and Kim CG. 2003. Genomic Cloning of Mud Loach Misgurnus mizolepis (Cypriniformes, Cobitidae) $\beta$-Actin Gene and Usefulness of Its Promoter Region for Fish Transgenesis. Marine Biotechnology 5:244-252.

Preston NP, Baule VJ, Leopold R, Henderling J, Atkinson PW, and Whyard S. 2000. Delivery of DNA to early embryos of the Kuruma prawn, Penaeus japonicus. Aquaculture 181:225-234.

Quitschke WW, Lin ZY, Deponti-Zilli L, and Paterson BM. 1989. The beta actin promoter. High levels of transcription depend upon a CCAAT binding factor. Journal of Biological Chemistry 264:9539-9546.

Rasmussen RS, and Morrissey MT. 2007. Biotechnology in Aquaculture: Transgenics and Polyploidy. Comprehensive Reviews in Food Science and Food Safety 6:2-16.

Saurabh S, and Sahoo PK. 2008. Major diseases and the defence mechanism in giant freshwater prawn, Macrobrachium rosenbergii (de Man). Proceedings of the National Academy of Sciences, India - Section B: Biological Sciences 78:103-121.

Shi Y, Soderlund M, Xiang J, and Lu Y. 2015. Function and regulation domains of a newly isolated putative $\beta$-actin promoter from pacific white shrimp. Plos One 10:517-528.

Shi Y, Xiang J, Zhou G, Ron TB, Tong H-I, Kang W, Sun S, and Lu Y. 2016. The Pacific White Shrimp $\beta$-actin Promoter: Functional Properties and the Potential Application for Transduction System Using Recombinant Baculovirus. Marine Biotechnology 18:349-358.

Sin FYT. 1997. Transgenic fish. Reviews in Fish Biology and Fisheries 7:417-441.

Zhou B, Long Y, Song G, Li Q, and Cui Z. 2015. Molecular characterization of the lgals1 gene in large scale loach Paramisgurnus dabryanus. Gene 577:65-74.

Zhu J, He FH, Hu SN, and Yu J. 2008. On the nature of human housekeeping genes. Trends in Genetics 24:481-484.

Zhu XJ, Dai ZM, Liu J, and Yang WJ. 2005. Actin gene in prawn, Macrobrachium rosenbergii: characteristics and differential tissue expression during embryonic development. Comparative Biochemistry \& Physiology Part B Biochemistry \& Molecular Biology 140:599-605. 


\section{Table $\mathbf{1}$ (on next page)}

Sequences for primers 
1 Table 1. Sequences of primers

\begin{tabular}{|c|c|c|}
\hline Primer name & Sequence (5'-3') & Note \\
\hline Mractb1-F & TTCCCATCCATTGTCGGCAG & cDNA amplification \\
\hline Mractb1-R & GCATTCTGTCAGCGATTCCTGG & cDNA amplification \\
\hline GSP1 & TGACCCATACCAACCATCACAC & 5'-RACE \\
\hline GSP2 & ACCGGAGCCATTGTCTACAACCAAC & 5'-RACE \\
\hline Exon1-F & ACTCGCTCTTCGACATC & cDNA cloning \\
\hline Exon1'-F & AGTCTGTCACTTGCTCC & cDNA cloning \\
\hline Exon3-R & TGTCTGTAGAAATGAATTTATTC & cDNA cloning \\
\hline Mractb1-SP1 & GTACTGATATGAAGGCGTGTTCAG & Genome walking \\
\hline Mractb1-SP2 & GATAATGATGGTAAGGCAAACATTG & Genome walking \\
\hline Mractb1-SP3 & TACACCTGGAGTGTCTAAGCAG & Genome walking \\
\hline Mractb1-SP4 & CTACTCCTGAAGATGTCGAAGAGCGAGTG & Genome walking \\
\hline Mractb1-SP5 & TAACATCTGAAATGAAAGCGGACGAAACTG & Genome walking \\
\hline Mractb1-SP6 & CAAACGTCTTGCCTTATATGGACATGGAG & Genome walking \\
\hline Mractb1-SP7 & GTAGAAAGACCGGGATTTCTTTCGGT & Genome walking \\
\hline Mractb1-SP8 & ACTGGGCGTAACTACTATGCCTCTAA & Genome walking \\
\hline Mractb1-SP9 & GTTGAAGGGAAATGTACTGAGAACA & Genome walking \\
\hline QMractb1-F1 & TCAGGAGTAGCACGTACAC & qPCR \\
\hline QMractb1-F1' & ATCACTGGTGCTCGTTG & $\mathrm{qPCR}$ \\
\hline QMractb1-R0 & ACAATGGATGGGAACAC & $\mathrm{qPCR}$ \\
\hline QMr18S-rRNA-F & TAGTTGGAGGTCAGTTCC & $\mathrm{qPCR}$ \\
\hline QMr18S-rRNA-R & ATTCCAGAGTAGCCTGC & $\mathrm{qPCR}$ \\
\hline Mractb1P1-F & ACTGAGCTCTCCCGAAGTGATCACTG & Promoter analysis \\
\hline Mractb1P1-R & AGACTGCTAGCTTTGTATTAGCTGCAAGAGAAAG & Promoter analysis \\
\hline Mractb1P2-F & ACTGAGCTCTCATTTAGTAAGTAGGAGAG & Promoter analysis \\
\hline Mractb1P2-R & 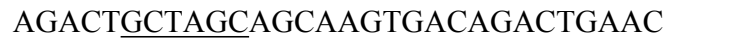 & Promoter analysis \\
\hline Mractb1P3-F & 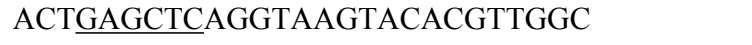 & Promoter analysis \\
\hline Mractb1P3-R & AGACTGCTAGCTGAGATAAATTATGGAAC & Promoter analysis \\
\hline Mractb1P4-F & 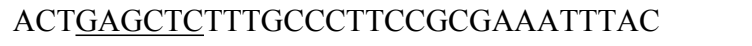 & Promoter analysis \\
\hline Mractb1P4-R & AGACTGCTAGCGGTGAGAGTGTACGTGCTAC & Promoter analysis \\
\hline Mractb1P5-F & ACTGAGCTCACATTATGGAAACATTTC & Promoter analysis \\
\hline Mractb1P5-R & AGACT $\underline{\text { ACTAGCATTCTTTGCATGTGACGAG }}$ & Promoter analysis \\
\hline Mractb1P6-F & 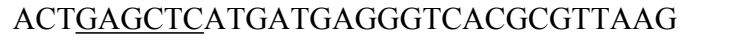 & Promoter analysis \\
\hline Mractb1P6-R & AGACTECTAGCTTCGGCGAACTGGGCGT & Promoter analysis \\
\hline Mractb1P7-F & 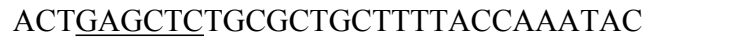 & Promoter analysis \\
\hline Mractb1P7-R & AGACTGCTAGCTCATCATCTTTACCATC & Promoter analysis \\
\hline Mractb1P42-F & TACACTCTCACCTCATTTAGTAAG & Promoter analysis \\
\hline Mractb1P24-R & CTTACTAAATGAGGTGAGAGTGTA & Promoter analysis \\
\hline Carpactb1-F1 & ACGAAGCTTTCAAACTGTGGCACCATC & Promoter analysis \\
\hline
\end{tabular}




\section{Figure 1}

Cloning of the 5'-flanking sequence of the Mractb1 gene

The $5^{\prime}$-flanking sequence of Mractb1 was cloned by three rounds of genome walking based on asymmetric interlaced PCR. The dashed line represents the assembled 5' flanking sequence. The rectangular blocks and the dotted arrows indicate the cloned sequences and the primers used for each round of genome walking respectively. The electrophoretograms were displayed accordingly. The numbers above the electrophoretograms indicate different round of PCR for each walking step.

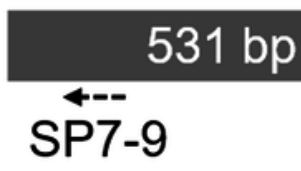

\section{$713 \mathrm{bp}$}
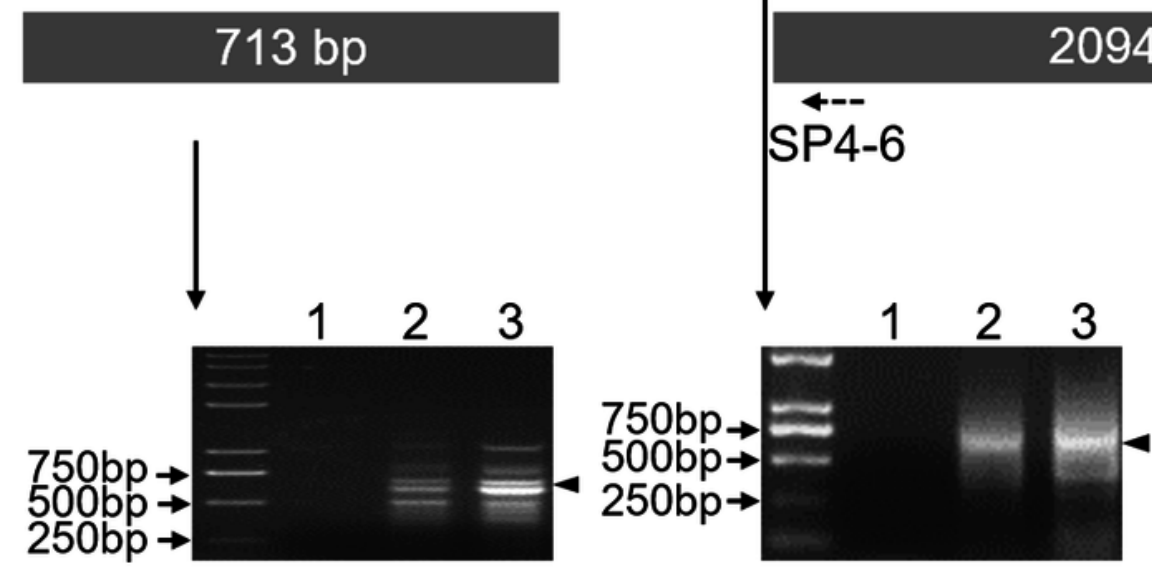

\section{4 bp}

\section{Known}

SP1-3

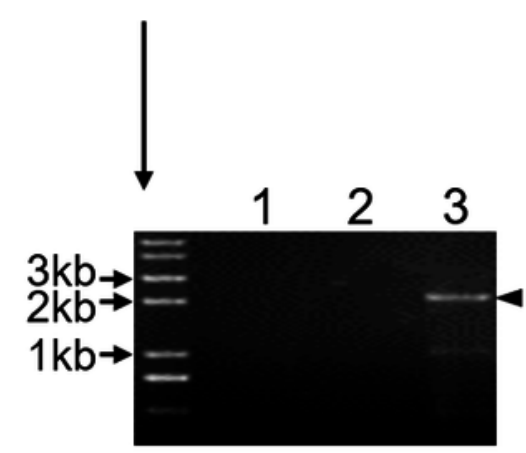




\section{Figure 2}

Determination of the transcription start sites of the Mractb1 gene

The shaded rectangles represent the known cDNA sequence (black) and the 5' cDNA end sequence obtained by 5' RACE (grey), respectively. The electrophoretogram shows the 5' RACE product (indicated by an arrow head). The base peak maps indicate the two 5' cDNA end sequences identified by DNA sequencing. M, DNA marker; GSP1 and GSP2, gene specific primers; In, 5' RACE inner primer.

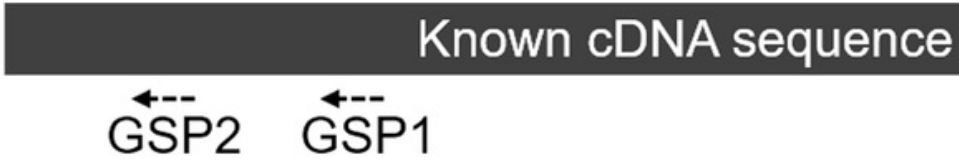

\section{5' cDNA end}

5' RACE Adaptor

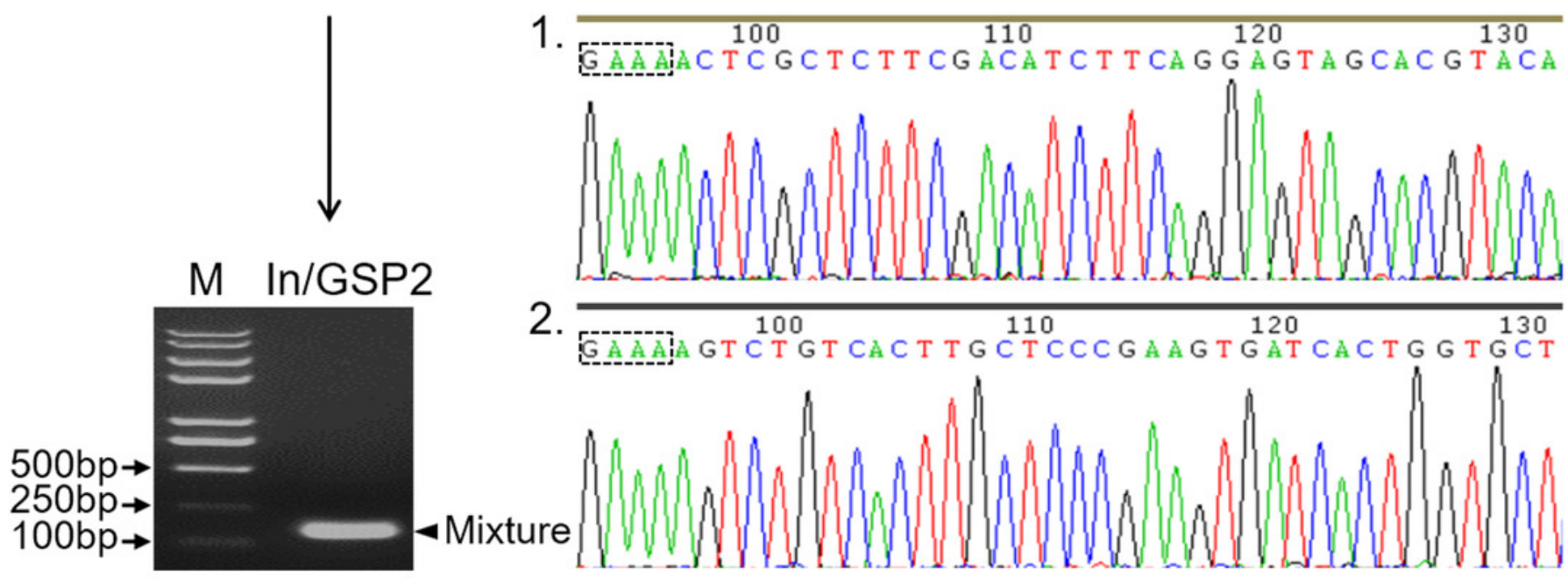




\section{Figure 3}

Transcripts of the Mractb1 gene

(A) Structure of the Mractb1 gene and its two transcript variants with different initial exon. The numbers and arrows indicate the relative position of the exons and coding sequence (CDS). The first base of the start codon was defined as position +1 . The numbers in the rectangles indicate different exons of the Mractbl gene. The alternative first exons are designated as exon 1 and exon 1'. (B) Electrophoretogram for the two transcripts. Primers used to amplify the two transcripts are Exon1-F, Exon1-F' and Exon3-R as displayed in (A). M: DNA marker.

A

Mractb1

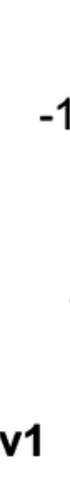

Driven by

Promoter-1

\section{Mractb1_tv2}

Driven by

Promoter-2

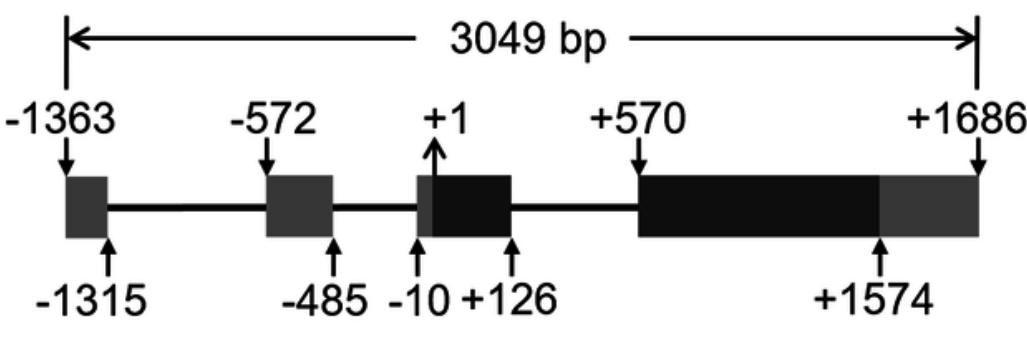

\section{Exon1-F}

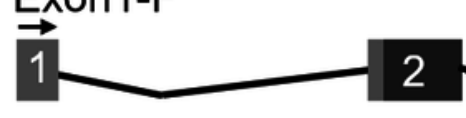

$\underset{\text { Exon1'-F }}{\rightarrow}$

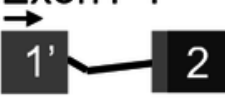

2
3

Exon3-
B

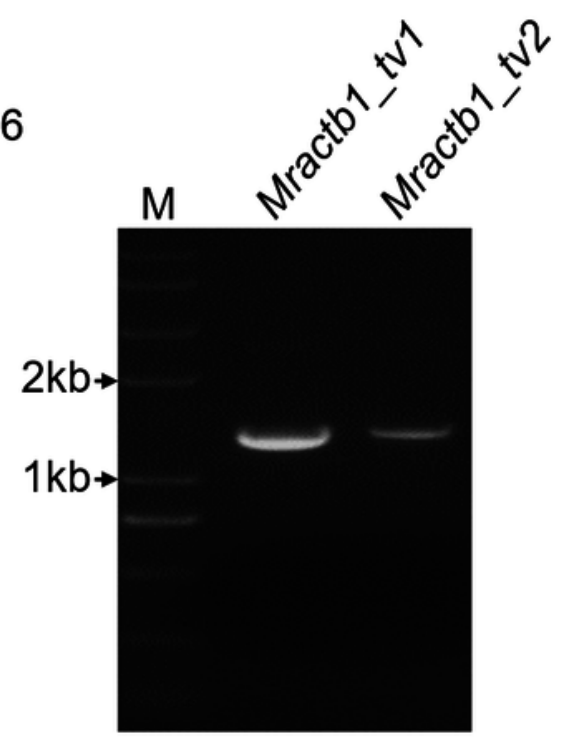


Figure 4

Expression of the two Mractb1 transcripts in different sexes and tissues
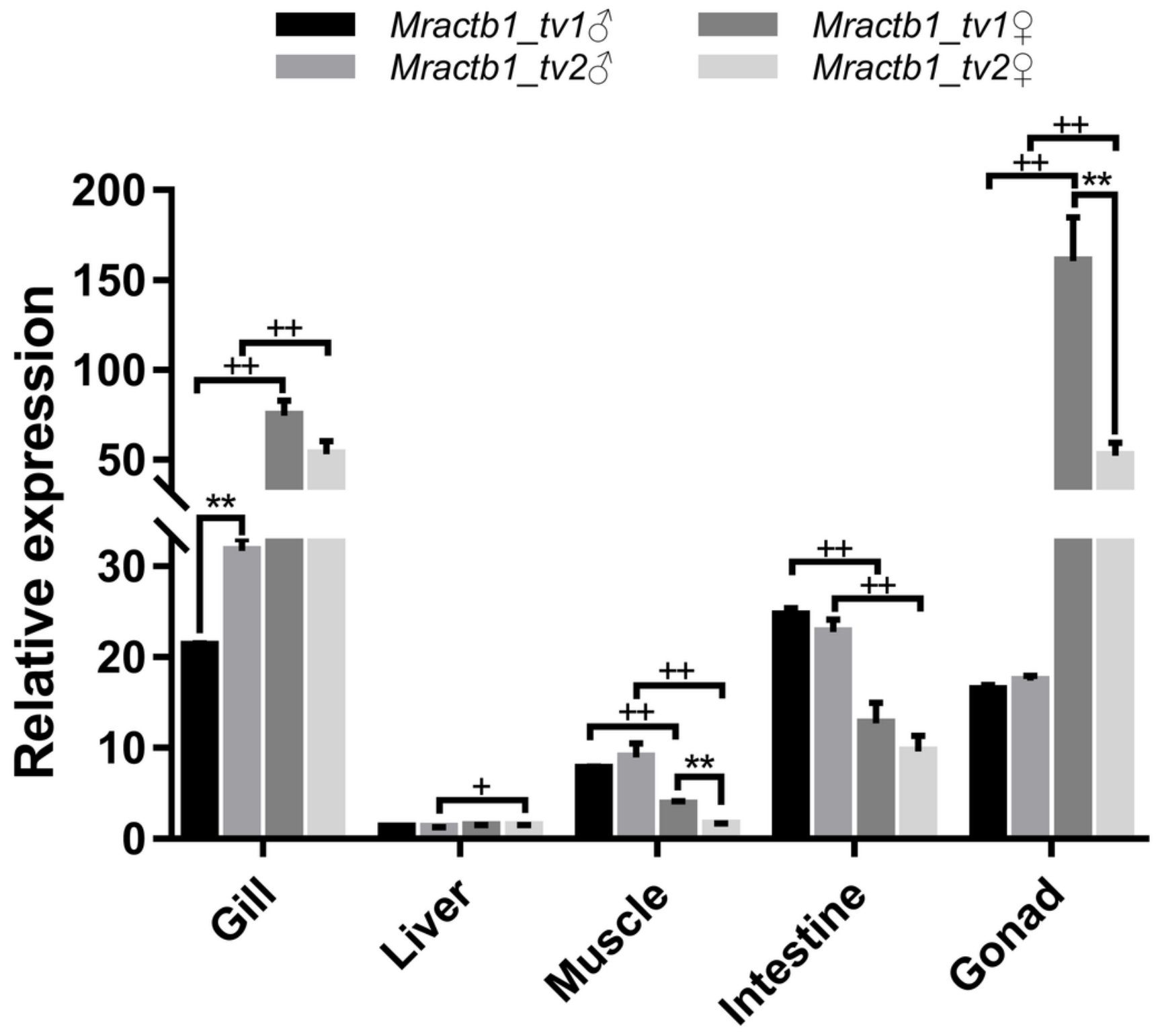


\section{Figure 5}

Molecular dissection of the Mractb1 gene promoter.

(A) Schematic diagram of the DNA fragments subjected to promoter activity assays. The 2620 bp 5'-flanking sequence was divided into 7 fragments (rectangles shaded with different color) according to the location of the TSSs and CpG islands (i1: -1762/-1569, i2: -1437/-1320, i3: -747/-542) predicted by the Methprimer software (http://www.urogene.org/). The arrow heads indicate TSSs. The dashed rectangles represent first exons of the two transcripts. (B) Promoter activity of the flanking regions characterized individually. (C) Characterization of promoter-1. (D) Characterization of promoter-2. (E) Deletion analysis of the whole sequence. (F) Interaction between the two alternative promoters. The DNA fragments were cloned into the pGL3-basic vector to generate promoter activity analyzing plasmids. The constructs were transfected into EPC cells and the transcriptional activities were analyzed by dual luciferase assays. The relative luciferase activity represents the ratio between the firefly luciferase activity and the corresponding renilla luciferase activity. The bars indicate mean \pm SD ( $n=$ 3). 


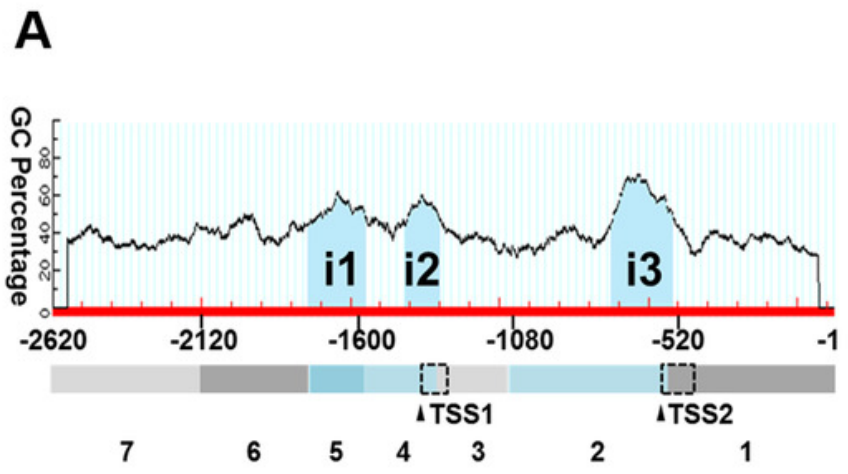

C
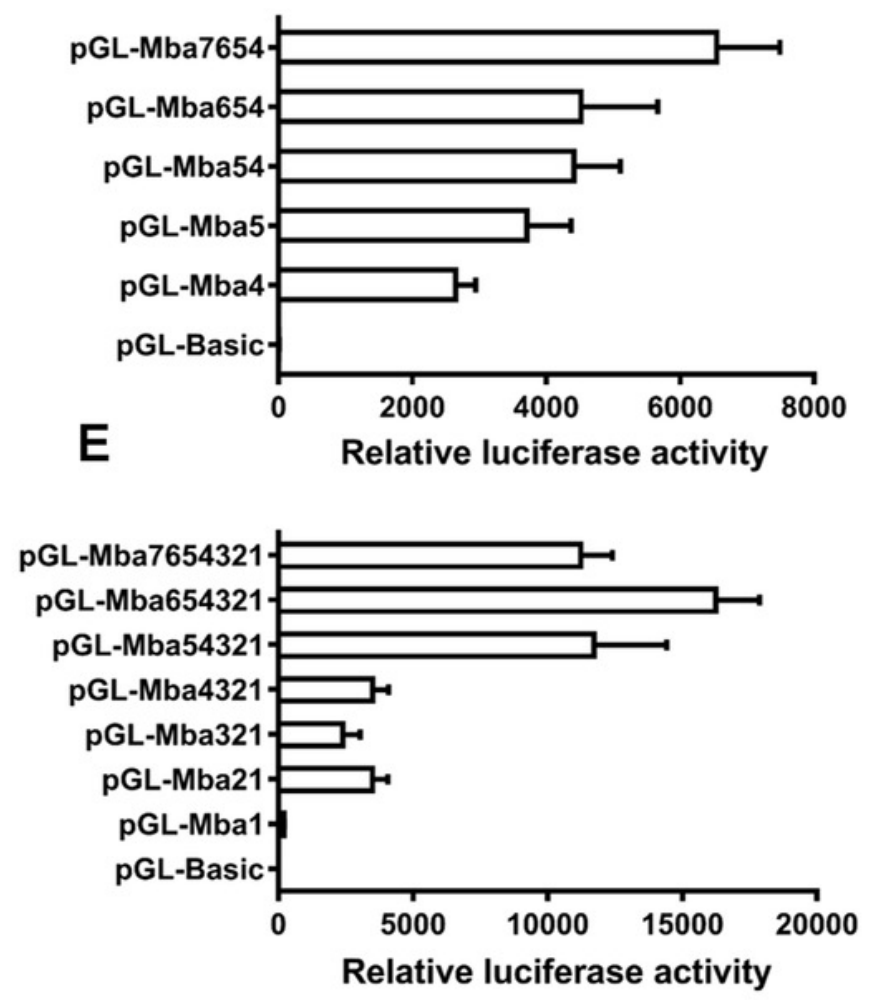

B

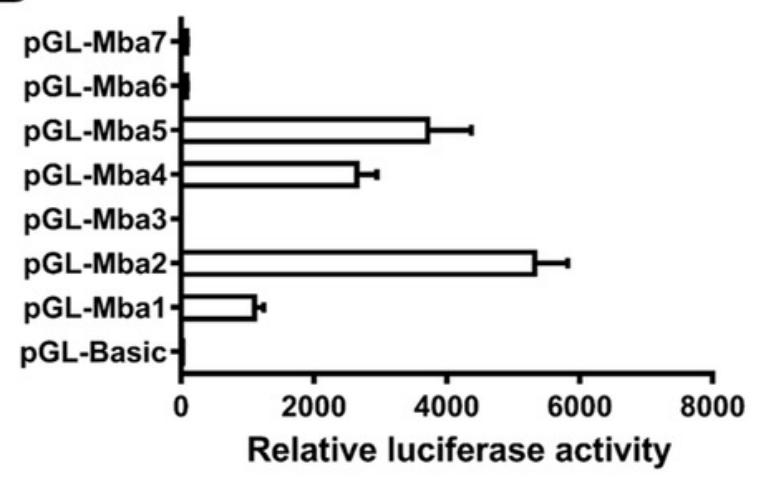

D
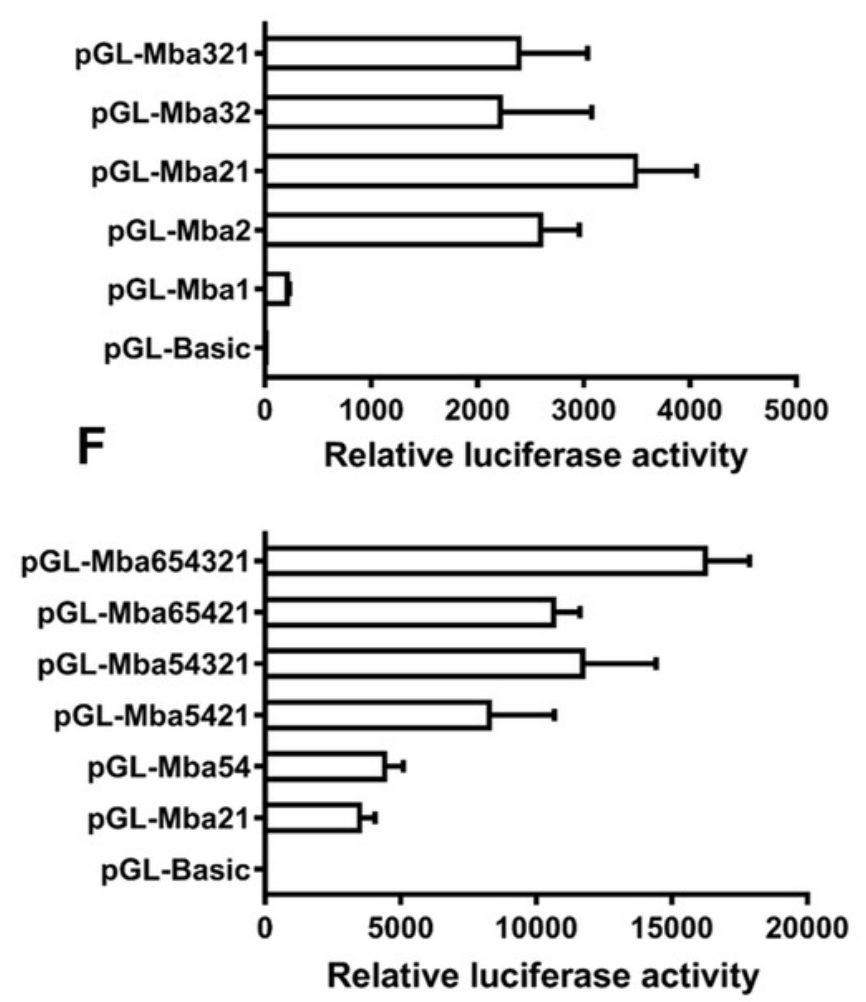


\section{Figure 6}

The conserved genetic elements in the Mractb1 5'-flanking region.

The conserved genetic elements in the Mractb1 5'-flanking region. The CpG islands are shown in red. The exons are shown in uppercase and the introns are shown in lowercase. The numbers at the left represent relative position of the nucleotides and the first base of the start codon is regarded as +1 . The donor/acceptor sites for splicing are shown in red italics. The arrows indicate transcription start sites. The CCAAT-box, CArG-box, GC-box and InR are underlined. 
-1766 a c a $t$ t a $t g g$ a

-1716 c c g c c t c g c a

$-1666$

$-1616 \mathrm{t} t \mathrm{~g} \mathrm{c} g \mathrm{~g} \mathrm{c}$ a a $a$

-1566 c c t t c c g c g a

-1516 a a $t$ c g c g a g g

-1466 c g g g a a a c t c

-1416 a a a g t c a g a g

$-1366$

$\overrightarrow{A C T C G C T C T T}$

InR

-1316 a a g t a c a c g t

$-1266 \mathrm{~g} \mathrm{~g}$ a a g a $\mathrm{t} t \mathrm{t} a$

-1216 a $t \mathrm{tg} \mathrm{g} \mathrm{t} \mathrm{t}$ a g t

-1166 a a $\mathrm{t} \mathrm{t} \mathrm{g} \mathrm{c} \mathrm{a} \mathrm{g} \mathrm{t} \mathrm{g}$

$-1116 \mathrm{~g} \mathrm{t}$ a $\mathrm{t} t$ a $\mathrm{t}$ a g a

-1066 g a g g c c g t g a

$-1016 \mathrm{t} t$ a g c c g g t $t$

-966 c g c g t a $t$ a a g

-916 c c c g a a a c c g

$-866 \mathrm{t}$ c c c c t c g t t

$-816 \mathrm{~g}$ a a $\mathrm{t}$ a $\mathrm{t} \mathrm{t} \mathrm{g} \mathrm{c} \mathrm{t}$

-766 c t t c t a a a a t

-716 t c a c g t g g c a

-666 c t c g a c g g c c

$-616 \mathrm{~g} \mathrm{c} \mathrm{g} \mathrm{g} \mathrm{g} \mathrm{g} \mathrm{c} \mathrm{c} \mathrm{c} \mathrm{t}$ box

-566 C T T GCT C C C G

-516 A T T T A T C A T

$-466 \mathrm{~g} \mathrm{t} g \mathrm{c}$ a g t $\mathrm{t}$ a

$-416 \mathrm{~g} \mathrm{t} g \mathrm{t} \mathrm{t} \mathrm{g} \mathrm{tg} \mathrm{t}$

-366 a $t$ c g t t g t g g

-316 g a a c $t$ t a c $t$

-266 a g t g t $t \mathrm{~g} \mathrm{~g}$ a $\mathrm{t}$

$-216 \mathrm{~g} \mathrm{t} g \mathrm{t}$ a a $\mathrm{t} \mathrm{t} \mathrm{g}$

-166 a c a c a t g t $t \mathrm{~g}$

-116 a a g g t $t$ t a g

-66 c c a a g c a g t g

-16 c a g C T A A T AC a a c a t t t c a a

$t \mathrm{t} c \mathrm{a} a \mathrm{cgt} c \mathrm{c}$

a $\mathrm{taggca}$ a g rG-box

a $\mathrm{t}$ a a $\mathrm{t}$ a g g g g

a a t t t a c g c c

$t \mathrm{t} t \mathrm{gtg} \mathrm{tc} a \mathrm{a}$

$\mathrm{g} \mathrm{g} \mathrm{tg} \mathrm{a} \mathrm{a} \mathrm{a} \mathrm{g} \mathrm{a} \mathrm{t}$

a g a c g t c g g

C G A C A T C T T C

$\operatorname{tggctgttac}$

$\mathrm{tg}$ a $\mathrm{tg} \mathrm{ggt} \mathrm{g}$

$t \mathrm{t} t \mathrm{tg} g \mathrm{~g} \mathrm{t}$

$\mathrm{tg} \mathrm{t}$ a $\mathrm{tg}$ a g t

$g \mathrm{t} a \mathrm{t}$ a $\mathrm{t}$ a g t

$t \mathrm{t} t \mathrm{t} t \mathrm{a} a \mathrm{t} a$

a $t \mathrm{tgtgtat} g$

$\mathrm{t}$ a a a g a a a $\mathrm{c}$

c c a t t g a a c a

a a g t $t$ a $t \mathrm{t} \mathrm{t} t$

$g t t t t g g g a$

$\mathrm{g}$ a a $\mathrm{a} g$ a c $t \mathrm{cg}$

a $t c t g g c g ~ t$

$t c a c c g c c t$

t t a t a t g c t a

A A G T G A T C A C

T A T C T G T G A T

a ga $\mathrm{t}$ a $\mathrm{t}$ a a c $\mathrm{t}$

$\mathrm{g}$ a a $\mathrm{a}$ c g a t g t

$t \mathrm{t} t \mathrm{a} a \mathrm{c} t \mathrm{t} \mathrm{tg}$

$\mathrm{tgc}$ a c a c t g g a c a g g t $t \mathrm{tcg}$

$t \mathrm{tgccggat} t$

a a g c t t a t t $t$

a c c t g a a t $t$

$\mathrm{tg} t \mathrm{t}$ a t t t t

A A A A T G c c g a a a ga a $t$ c c c g g t c t t

t g c t c gac c a a t cagcg g c c CCAAT-box

a c g t t $\mathrm{tg} \mathrm{t}_{\mathrm{c}} \mathrm{t} \mathrm{gggcg} g \mathrm{ggcc}$ GC-box

a g t $t c a c t c g \quad t c a c a t g c a a$

$g c a g t t t c g t \quad c c g c t t t c a t$

c g g g t c a t c g t g a a t a t t c

a a a g t g a $t$ a $t$

a t $t$ g c c t c c

t g t $t$ tg gag $t$

$\mathrm{g} \mathrm{g} \mathrm{c} \mathrm{g} \mathrm{c} \mathrm{g} \mathrm{c} \mathrm{t} \mathrm{g} \mathrm{c}$

A G A A T A C A C C T ACACTCT

c a a g t a t c t $t$

$\mathrm{t} t \mathrm{gtg} \mathrm{t}$ a g a g

t a $\mathrm{tg} g$ a a g g c

a $t \mathrm{tg} g \mathrm{t} \mathrm{tg} \mathrm{g}$

$t c c$ a $t$ a a $t$ t

t g c t t t t a a

$g t t a g t$ a g t

$t \mathrm{tgct} t \mathrm{t} c \mathrm{c}$

a t g t a c t t $\mathrm{tc}$

c a c c a g a g t c

$\mathrm{g}$ a a t $t \mathrm{t} t \mathrm{t} \mathrm{g}$

t g g t $t$ a g g g a

$c$ a c g g g g c t g

a c c a c c t c a t

$g g g c c g t t c c$

T G G T G C T C G T

A A T A T C T G gt

$\mathrm{t} t \mathrm{t}$ a c t $\mathrm{tg} \mathrm{t} g$

g c c a c c a a c c

$\mathrm{t}$ a $\mathrm{t}$ a $\mathrm{t}$ a a a $\mathrm{t} \mathrm{t}$

c a t g t a a a g t

a t t c c t t a g a

$t c g a g g c t c c$

$\mathrm{tg}$ a a a c t t g

$c$ a t $\mathrm{tg}$ a $\mathrm{tg}$ a

a a a t t a c t t c g t c a a tg t g

$g \mathrm{t} a \mathrm{ggt} t \mathrm{t}$

$t \quad c a g g c t ~ t a$

$t \mathrm{t}$ a $\mathrm{t}$ t g c a a

a t c t c a t t t a

c c c c a g t $t$ a $t$

$\mathrm{t}$ a a $\mathrm{t}$ a c a g t c

a a c c a t a a g t

a c c g g t a a c t

g t $t$ t a a t g a

a a g t c t g a g a

$\mathrm{gtg} \mathrm{t} c$ a a g c g

a c a c c c c c c c

$\mathrm{tg}$ a c g g t g g a

$g \mathrm{gtg} t \mathrm{ggt}$ CAGTCTGTCA

T G G G C C T C T T

a a a $\mathrm{t}$ a $\mathrm{t} c \mathrm{t} \mathrm{tg}$

a a a t t t c t g

g g t t $\mathrm{gg}$ a a $\mathrm{g}$

a $t$ a t t g a c a

$\mathrm{g}$ a a $\mathrm{tg} \mathrm{t} \mathrm{ct}$ a g

a t c c t t c t g a

$t a g c t t t t g$

a a g c t t a g t t

$\mathrm{gtt} t \mathrm{tactca}$

a a c a c t a t t a $t c t a c t a t t c$ $\mathrm{g}$ a g a $\mathrm{tcgtct}$

$\mathrm{g} a \mathrm{c} \mathrm{g}$ a g g c c a

a g a a $t \mathrm{t} \mathrm{t} \mathrm{gc}$ $t \mathrm{t} c$ a g a $\mathrm{tg} t \mathrm{t}$ c t t t a a t a t a c c c c a c c t g g $\mathrm{tcct} t \mathrm{ctctc}$

C A C C T G A g $t$

$t$ a g t t t $t$ a $t$ c g t t t g g a t a c t t g a t $\mathrm{t}$ a g a g t g t c g a g a $\mathrm{g} \mathrm{t}$ a a g t a g ga $t c t t$ a a a c a t t t t a c a t $\mathrm{t}$ a g g t c a c t $t$ $t \mathrm{t} a \mathrm{ctc} c \mathrm{t}$ c c gat $t \mathrm{t}$ g t c g a t a a a a $\mathrm{c}$ $c$ a t g g t g a c g c a c c c c c c c a $\mathrm{g} \mathrm{g} \mathrm{a} \mathrm{a} \mathrm{a} \mathrm{g} \mathrm{g} \mathrm{g} \mathrm{g}$ InR

G T C C C A T T C C

$\mathrm{tg} g \mathrm{t} t \mathrm{t} t \mathrm{t} t$

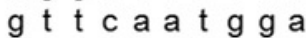
$\mathrm{t} \mathrm{tg} \mathrm{tgatg} \mathrm{g} \mathrm{c}$ a c t t t g g a $\mathrm{tg}$ c a t t ga t t t c a c a a $t \mathrm{t} t \mathrm{t} \mathrm{t}$ a c c a t c a a a t $\mathrm{tg} a \mathrm{a}$ a $\mathrm{gct} \mathrm{tg}$ a g t t t a t a t $c t t c t c t t g$ 


\section{Figure 7}

Mractb1 promoter demonstrates comparative activity with carp actb1 gene promoter.

(A) Relative luciferase activity. (B) Western blot of firefly luciferase. (C) Relative levels of the firefly luciferase protein. The Mractb1 promoter (Mbap) and carp actb1 promoter (Cbap) were cloned into the pGL3-basic vector and the resulted constructs were transfected into EPC cells. Dual luciferase assays and western blot were performed to analyze the activities and protein levels of the luciferase reporter, respectively. GAPDH was used as loading control for western blot. The relative intensity represents ratio of the brightness of the firefly luciferase bands to those of GAPDH. The bars indicate mean \pm SD $(n=3)$.

\section{A}

C
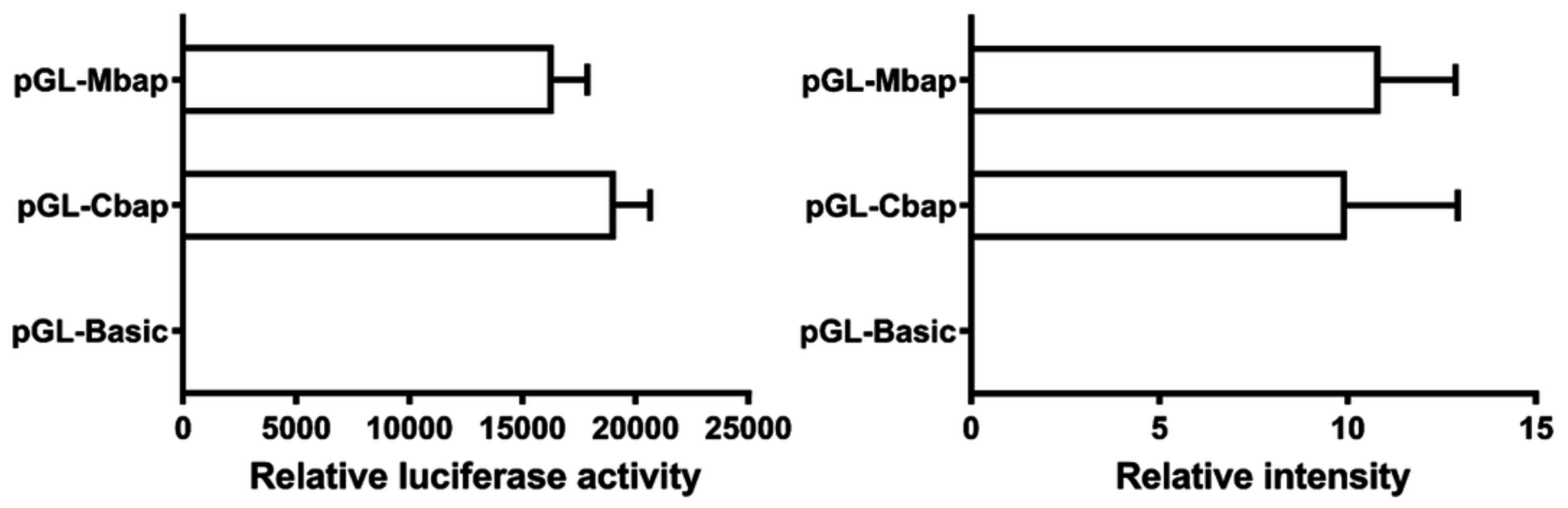

B

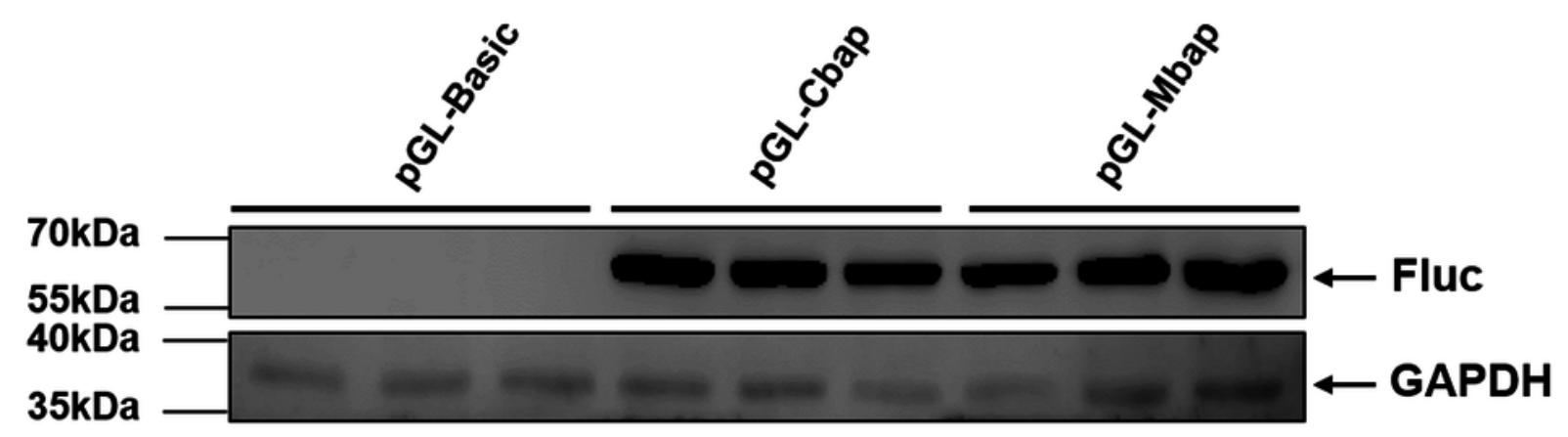

